that he has cut the hepatic artery. It is worth remembering that the surface of the liver, stripped by the removal of a simple mucocele, will often bleed more freely than after the removal of an infected gall bladder. When the detachment exposes a wide area of the liver, bile sometimes leaks from small bile ducts thus damaged, sometimes so freely for twenty-four hours or more as to raise the suspicion that the ligature has slipped off the cystic duct.

\section{Complations of Cholecystectomy.}

An important complication of calculous chulccrstitis is fistulous union of the gall bladder and colon due to ulceration and escape of gall stones into the gut. A gall stone which slips into the colon in this way may be as big as a bantam's egg. The museum of St. Mary's Hospital, London, contains a segment of pelvic colon with a gall stone of this size impacted in it, and with fatal consequences. Many stones escape through fistulous openings in the gastrointestinal tract, to the permanent advantage of the patient. The orifice of escape in such a complication shrinks to a narrow funnel and this occasionally resembles a cord. In dealing with apparently solid cords a ligature should carefully be applied; a narrow chanuel connumicating with the gut may exist in it and be sufficient to set up local sepsis, illustrating the maxim-a small leak neglected may sink a great ship.

Nothing seems more surely established in clinical observation than the idea that complete obstruction of the eommon bile duct is inevitably followed by jaundice. A spinster, aged 50, complained of a pain in the region of the gall bladder. She was thin and I could feel a gall stone in the position of the common bile duct. There was neither sign nor history of jaundice. I removed a large stone from the common duct which had completely jlugged it (Fig. 3). The gall bladder adhered to and commurieated freely with the transverse colon. In this woman the bile found its way to the gall bladder by the cystic duct and dribbled through the fistula into the gut, to the patient's temporary advantage. As the removal of the stone reopened the natural outlet for the bile, I removed the gall bladder and closed the hole in the colon. The woman reported herself in good condition ten years afterwards.

The gall bladder is a diverticulum of the hepatic duct. The mere loss of the gall bladder is unattended with sinister consequences. Many mammals and birds lack a gall bladder. Semo parrots have a gall bladder and some not. Hamlet, in his soliloquy on the First Player, says:

But I am pigeon-lirer'd and lack gall To make oppression bitter.

Act $\mathrm{ii}, 2,605$.

This clearly indicates Shakespeare knew that the pigeon lacks a gall bladder. Absence of the gall blad:lor neither causes inconvenience nor shortens life. In 1906 I removed the gall bladder of a widow, aged 76, for acuts calculous cholecystitis. The mucous membrane of the gall bladder was thickly beset with flakes of cholesterol and glistened like the tinselled coat of Harlequin. The patient lives, aged 94, and bids fair to become a centenarian.

\section{Radiogriphy and Gall Stontes.}

One of the difficulties in persuading patients to submit to operative treatment for suspected gall stones was uncertainty of diagnosis. "Give us a sign" was the imperative and persistent demand. Many physicians preferred to trust soap pills and olive oil, rather than the seajpel. The position has changed. Radiography can secure shadows on photographic plates which indicate not only the presence of gall stones, but their size and shape. With such evidence patients now willingly submit to surgery; and, as a rule, quick healing, freedom from leakare, and from the recurrence of gall stones, with quick restoration to health and usefulness, are the usual consequences of cholecrstectomy. To operate for acute cholecystitis and not rimove the gall bladder is as reprehensible as operating for gangrenous appendicitis and leaving the appendix.

The golden rule for surgeons to observe is this: Examine the main ducts-cystic, common, and hepatic-especially the ampulla, and be satisfied they are free of concretions, remembering always: $A n$ undiscovered concretion in the common duct will wreck a cholecystectomy. The manifestation may be long delayed, but it will surely come. Although the medicine-man can point to some wonderful cures, and sorcerers can cast out imaginary devils, neither a sorcerer nor a physician can expel gall stones.

\section{A ON}

\section{LUMBO-SACRAL BACKACHE.}

P. JENNER VERRALL, F.R.C.S.,

ORTHOPAEDIC SURGEON, MINISTRY OF PENSIONS HOSPITAL, SHEPHERD'S BUSII, AND NORTHCOURT HOSPITAL FOR CHILDREN ; CHIEF ASSISTANT, ORTHOPAEDIC DEPARTMENT, ST. BARTHOLOMEW'S HOSPITAL.

Lumbo-sacril pain is exceedingly common, very disabling, and often so resistant to treatment that patients will resign themselves to it. Either its treatment devolves on the general practitioner entirely, or the patients, if they can afford it, go from specialist to specialist in search of relief. We have often to deal with a vicious circle inrolving many systems, but with only one symptom-pain. This pain may be situated in various positions, may or may not be affected by jarring of the spine, standing, or walking, and may be variously described as sharp, gnawing, or aching. It is of the first importance in diagnosis to obtain an accurate account of this symptom from the patient. We may simplify the subject by dividing the possible causes into five groups, three of which, the most serious, will not detain us long, but must be carefully excluded before wo consider the other more common causes. We have to deal with a complex system of bones, joints, ligaments, muscles, and nerves, but we may separate the sacro-iliac joints for consideration later.

\section{Severe Bone and Joint Discase.}

Caries of the lumbar spine is not uncommon, and it must be remembered that adults are by no means exempt and that caries does not necessarily affect the bodies of the vertebrae, but may affect, though less commonly, the laminae, transverse processes, etc. Diagnosis will be made by consideration of the extreme rigidity, possible deformity and abscess formation, and by the $x$-ray findings. Spinal skiagraphy is by no means easy, and in doubtful cases it is essential to have a skiagram by an expert expertly interpreted. The possibility of gummatous deposits in the spine must not be forgotten; the symptoms resemble those of tuberculous caries, and the two diseases may ccexist. Syphilis of the spine is, however, a rarity, and the same mar be said of sarcoma; but secondary carcinoma, frequently associated with a mammary tumour (possibly unsuspected), is by no means uncommon. Forewarned is forearmed, and severe localized pain coming on in a patient liable to carcinoma will lead to an adequate $x$-ray examination, when diagnosis is usually easy, as the skiagraphic appearances are generally characteristic. Aneurysm and acute osteomyelitis are, of course, exceedingly rare, but we must not forget them. I do not propose to enter into the ireatment of these cases as this would take us too far.

\section{Arthritis of the Lumbar Spine.}

Here again the skiagram affords us the only absolutely sure method of diagnosis. Lipping of the edges of the rertebral bodies and osteophycic outgrowths are characteristic. These latter may even go to the extent of complete fusion, producing actual bony ankylosis between the rertebrae. Serere arthritis (spondylitis deformans), with its flat absolutely rigid spine, is fortunately rare, as treatment can achiere little more than the arrest of the disease when it is well advanced, early diagnosis being the only hope. Gonorrhoea is responsible for at least 65 per cent. of such cases. Some surgeons would put the percentage bigher, but quite 30 per cent. are due to metastatic infection from

* Delivered before the Winchester Division on April 9th, 1924. 
the intestinal tract (dysentery, typhoid, and even Bacillus coli), and this quite apart from the acute or subacute spondylitis not uncommon during the later stages of such diseases, and of less serious prognosis if correctly treated. Spondylitis of this type is often associated with iritis, both in the gonococcic variety and in the intestinal; the infective agent in the latter is either the primary organism or, more commonly, a streptococcus which has reached the system through the open intestinal ulcers. Rarely, perhaps in 5 per cent., spondylitis deformans is due to metastases from other septic foci. Milder degrees of arthritis of metastatic origin are common. Moderate rigidity and pain in the lumbar spine combined with intermittent attacks of lumbago are associated with such arthritis. It must not be assumed that every case of lumbago has arthritis, as the affection may be a pure.fibrositis; but arthritis is sufficiently frequent to justify a skiagram, which will often reveal either slight lipping of the vertebral bodies or quite as commonly osteophytic outgrowth in some of the interneural joints.

Treatment will follow the usual lines as for arthritis elsewhere. In the acute stage rest is imperative, either on a frame or plaster bed in severe cases, or, in mild cases, simply in bed with the lumbar spine supported by a pillow. Where abnormal curves are in course of development, an attempt should be made to correct these by suitable padding of the bed or frame during the period of recumbency. It would be absurd to condemn every patient suffering from slight spinal arthritis to permanent spinal fixation. Radiant heat, counter-irritation, and ionization may suffice for mild eases. Subacute cases may need firm fixation in a plaster jacket for a time, but in chronic cases where the disease is sufficiently extensive to justify fixation the ordinary Thomas's back brace will meet all requirements, and patients will be so much relieved by wearing it that they will not complain of the necessary fixation of the spine. While carrying out this local treatment a rigorous search must be made for the toxic cause, bacterial or chemical, and the appropriate treatment applied.

\section{Traumatic.}

Arthritis is often of traumatic origin, a relatively mild trauma providing a point of diminished resistance to the attack of the circulating toxin, as so frequently occurs in the joints of the extremities. But we may have more severe lesions. A history of violence, direct or indirect, will lead to the necessary investigation. There may be lesions of bone, ligaments, or muscles, or all together, or simply a concussion.

Of the grosser lesions, crush fractures of the vertebral bodies are commoner than is usually thought, and we must not be blinded to the possibility of their occurrence by a history of comparatively slight immediate disability after the accident, as the fracture is impacted and there is relatirely littlo instability. If undiagnosed, such fractures will often yield gradually to the body weight and a deformity will appear with the necessary concomitant symptoms of spinal distortion. In young subjects considerable violence is necessary, and the war provided us with many examples in men who had been blown up and buried under heavy masses of earth; and such accidents occur also in civil life. In older patients, however, far less violence is needed. I have a patient, a lady of 65 , who sustained a severe crush fracture of her first lumbar vertebra by stumbling when getting out of bed and falling with her back across a chair. Minor fractures of laminae and articular processes arise not infrequently from indirect or direct violence, often during athletic pursuits. Diagnosis is all-important, and the skiagram should ensure this when there is any doubt.

Treatment in the initial stages is by rest and complete fixation of the spine, and the prognosis is good, firm union usually occurring in three or four months. Subsequent symptoms will be due either to vertebral distortion, if such has been allowed to occur, or to adhesions, either in the joints themselves or round the exits of the spinal nerves. Such adhesions are not always preventable, but much may be done by instituting exercises at the correct moment. It is not easy to judge this moment accurately, but symptoms and the skiagram will, with experience, be sufficient guide. In old-standing cases, where the skiagram shews firm union, it is quite correct treatment to break down adhesions by mobilization and to follow this up with active exercises.

Spondylolisthesis must be mentioned, but is very rare. It may occur either gradually or by sudden violence. The skiagram of such a case shows a dislocation of the lumbar spine on the sacrum, causing a noticeable hollow in the back above the sacrum. The skiagraphic appearance in this case is unmistakable, the antero-posterior view showing the round outline of the vertebral body, and the lateral view (if obtainable) the dislocation forwards. Apar's from the well known obstetrical considerations, such cases, if they have symptoms or a progressive deformity, must be treated with a firm back brace with adequate abdominal support.

Sprains of the spine are common, especially in those engaged in athletics. Obviously many structures are involved together, and a more or less accurate diagnosis is obtainable only by a study of the symptoms produced by movement in various directions. It is hardly necessary to add that such movements must be made with extreme care, owing to the pain caused and to the possibility of the injury being more severe than one had imagined. A skiagram should always be taken if circumstances permit. Treatment consists in rest at first, and fixation, either by strapping in mild cases or by plaster in severe ones. When the acute symptoms have passed off, local massage will aid absorption of effusion and expedite repair, and graduated active exercises should be instituted at the earliest date consistent with safety. This latter is essential if adhesions are to be prevented, but in practice is very difficult to control, and it may well happen that adhesions form, and there may be pressure round the exits of spinal nerves with resultant neuralgia. Fortunately can easily recover the lost ground, when all acute symptoms are passed, by mobilization of the spine followed by exercises.

Mobilization to be effective must be thorough, and the method described by Sir Robert Jones ${ }^{1}$ represents the usual practice of orthopaedic surgeons. Nitrous oxide anaesthesia is sufficient for the mobilization of the minor joints, but it does not provide adequate relaxation for the treatment of the shoulder, hip, or spine. A full anaesthetic is given and the knees and hips fully flexed so as to give control of the pelvis. Then, while an assistant steadies the shoulders, the pelvis is held, and the spinal movements of flexion, extension, lateral flexion, and rotation are performed seriatim to the full degree. Mobilization must always be followed by active supervised exercises, commencing on the day following the operation.

I have said that trauma may cause concussion without an organic lesion. I refer to the familiar railway spine of the textbooks. The spine may be held absolutely rigid and the symptoms may be pronounced, but momentary relaxation by subterfuge may often be obtained. Characteristic spots of tenderness to pressure will usually be found on either side of the spine opposite the exits of the spinal nerves, the patient will be evidently neurasthenic and inclined to exaggerate symptoms, and finally the skiagram will be negative. I do not wish to dwell long on this sub. ject; suffice it to say that, when the legal aspect of the matter has been settled, mobilization will have both a moral and physical effect, especially if combined with the application of a cautery (or even simply a scrubbing-brush) to the back, and an assurance of cure. Akin to this is the hysterical spine, characterized by the same tender spots and often a fantastic attitude. I will not presume to encroach on the domain of psychological medicirc, except to remind you that such cases are often due to mimicry of a genuinely diseased acquaintance. Whethor treated by the orthopaedic surgeon or by the psychologist suggestion is the clue to treatment. The surgeon will combine this with mobilization and the psychologist perhaps with actual hypnotism.

Mechanical.

We now come to the commoner and more important causes. The back is an exceedingly complex structure containing a large number of bones, joints, ligaments, and muscles, whuse functions are interdependent. 
First, what are the potential morements of the spine? Flexion and extension occur mainly in the lumbar spine ${ }^{1}$ and are those movements which mainly concern us at present. Side bending is mainly lumbar in the erect position. In flexion it occurs rather higher and in hyperextension lower. Side bending and rotation are Jargely combined, rotation, however, being more a dorsal function. It is well to remember that in the flexed spine the dorsal bodies rotate to the convex side of the curve and in the extended spine the lumbar bodies rotate to the concave side, the result being that dorsal rotation is greater and lumbar rotation less than an examination of the spinous processes would lead us to suspect. One must also bear in mind the normal curves of the spine and their method of development. The primitive foetal type is an antero-posterior continuous C curve. The cervical lordosis commences to form soon after birth and the lumbar at about 6 months, the former being complete at about 1 year, and the latter developing more gradually. The maintenance of these acquired curves depends on muscular action, and any lack of muscular tone leads to a tendency to reversion to the foetal type with associated pain, from dragging on ligaments, etc., and from the eficrts made to prevent this reversion. This naturally brings us to a consideration of the effect of the muscles in maintaining position. The erect posture is maintained by balance combined with the integrity of the postural contraction of the essential muscles. For a thorough unde:standing of this postural contraction we are indebted to the researches of Sherrington and to the application of his results to orthopaedic subjects admirably set forth by Bankart. ${ }^{2}$ To summarize his conclusions briefly: The contraction of muscles is of two kinds-phasic, concerned with movement, and postural with the maintenance of posture. Those muscles which normally serve to maintain the erect attitude are capable of either of these two functions, and there is reason to believe that the two functions are controlled by separate innervations, that of postural contraction passing through the sympathetio chain.

In normal postural contraction no fatigue is caused, and the musele has the peculiarly converient quality of being capable of adapting itself to different lengths without change of tension. Ultimate control comes from the paracerebellar nuclei, which are in intimate connexion with the higher centres and with the semicircular canals.

Although we have seen that fatigue does not normally occur, very slight causes suffice to upset this balance and voluntary muscular action must be called to assist. Neurasthenia, adenoids, eyestrain, and excessive mental work are merely some of the causes of this. You are all familiar with the child who is devoted to books, works hard at homework, and generally gets prizes, but who complains of backache and has a kypholordosis. No exercises will cure such a child; the muscles are not weak, but their control is insufficient and we must treat the cause. Bankart has made the important observation that in such cases the myasthenic reaction is present, which is conclusive proof of the truth of the above. What is true of such a child is true also in older people, and a search for a cause of lack of nerve control, toxic or mechanical, will frequently enable us to cure an obstinate backache. We must also remember that in the aged there is normally an obliteration of the acquired curves and a reversion to foetal type, combined with decreased postural activity from general weakness.

The immediate effect of such lack of adequately balanced muscular control is that the spine is left unsupported, and open to the influence of outside factors. Habitual posture alone will not influence the spine, but when resistance to distortion is absent it will do so. The result may be a scoliosis, quite pliable and easily corrected in the early stages. Diagnosis is easy; inspection of the back will reveal the curre. Pain will be chiefly complained of on the convex side owing to excessive strain on ligaments and to increased voluntary muscular effort and consequent fatigue. There is frequently. an actual acute lumbo-sacral angle which produces a concentration of pain in the lumbo-sacral region. This angle is usually obvious, but a skiagram will detect it with greater certainty. The importance of its recognition lies in the necessity for correcting it before correction of the superincumbent spine is attempted. On the other hand, the spine may yield in an antero-posterior direction and cause pain.

No description of these defects would be complete without reference to Goldthwait's types. ${ }^{1}$ This very careful and accurate American observer has described two trpes of pérson particularly liable to suffer from lumbo-sacral backache, and most people will agree with him. His first trpe, termed by him the visceroptotic, has a long narrow back, flexible spine, and increased lordosis, with a sharp angle at the lumbo-sacral junction. The effect of this is obviously to produce an increased strain at the sacro-iliac joints, and pain is located there. His second type is the reverse of this-a thick-set body with slight lordosis and little movement of the lumbar spine. Here the strain is thrown on the lumbo-sacral angle.

The abdomen of a quadruped is supported directly from the vertebral column by muscles aided by a certain amount of elastic tissue. The adoption of the erect attitude in man has necessitated a different mechanism, and a lordosis is developed. This serves to balance the body, and at the same time to afford some support to the liver. Nature also anticipates intermittent increases in weight in the human abdomen. The most important of these is that of the gravid uterus, but food in the intestine and increased adipose tissue on the abdominal wall are also provided for. Pregnancy is a normal function, and the various necessary alterations are so marvellously successful that we should expect no ill effects. The savage woman develops increased lordosis and carries on her normal work without complaint; modern civilized woman develops the lordosis, but her overstrained nervous system refuses the postural contraction of the erector spinae, and she must use voluntary effort with consequent fatigue and pain at the lower attachment of the erector spinae. The wearing of a pregnancy belt is the price she pays for civilization. Pregnancy is, after all, a normal function, but overloaded intestines and an abdominal fat pad are not. Actual enteroptosis may be added to this, and the resulting disturbance of the centre of gravity is great. At the same time there is no natural tendency to compensate for this by increased muscle power and tono in the back; on the contrary, there will be toxicity and weakness. At first voluntary effort will be required, with consequent muscle strain, effort, and fatigue. When this fails all the strain will be thrown on the ligaments, with resulting pain from this cause. This is perhaps the commonest cause of lumbo-sacral backachic, the pain being felt mainly over the lumbo-sacral attachnient of the erector spinae. On the other hand, we may find a diminished lordosis with pain due to perpetual ligamentous strain without adequate muscular support. In those women who are compelled by their social status to undertake heavy domestic duties a common cause of backache is to be found in the low wash-tub and low kitchen sink. Perpetual stooping over these when tired throws an impossible strain on the erector spinae, lordosis diminishes, and backacho is constant. The remedy is obvious. At the other end of the social scale, modern fashion has decreed that women shall have no hips, a flat back, no mammary development, and a head bent forward. In scientific language we may describe this as a deliberate reversion to the foetal trpe of spine with obliteration of the normally acquired curves. As modern life must of necessity throw a large strain on the nervous system of such women, we cannot expect any great degree of postural activity in their muscles. The result is inevitable. When once voluntary action becomes fatigued pain must and does follow, while the necessary subsequent redevelopment of the normal curves is naturally associated with additional pain from ligamentous strain. Against the dictates of fashion the doctor is powerless, but he can do his duty and advise, and even if his advice be neglected for the moment it may be appreciated later on.

Abncrmal spinal curves may be due also to outside mechanical causes. A large number of people have one leg shorter than the other. The vast majority of these develop no deformity and no symptoms, but a pelvic tilt must exist, and where symptoms do occur we must notice and correct it by a high boot. Increased lordosis may be associated with genu valgum and flat-foot in which there is internal rotation at the hip and a consequently inclined pelvis. 
Similarly in talipes equinus there is pelvic tilting from insufficiency of the posterior structures of the leg. Such deformities will need appropriate correction.

\section{Sacro-iliac Joints.}

In England sacro-iliac disability is considered rare, but in America considerable attention is paid to these joints, and, I think, rightly. The characteristic symptoms are, first, pain at the site of the joint, increased by any jar on the limb of the affected side, such as stepping off a step, and a feeling of insecurity, which is easily understood when we remember that the whole weight of the body above this point must be transmitted through these joints. Examination will reveal deep tenderness over the joint (to be distinguished from the superficial tenderness due to referred pain) and pain elicited by lateral compression of the pelvic crests. These signs and symptoms are common in greater or lesser degree to all sacro-iliac troubles. Examination by $x$ rays is of doubtful value, as, although gross disease such as tubercle will be readily shown, milder arthritis and relaxation cannot be diagnosed by this means, as no accurate joint line is obtainable in the skiagram owing to the tortuosity of the joint. In tuberculosis, a very serious disease, symptoms will be marked; possibly there will be intra- or extra-pelvic abscess formation, and we shall be unlucky if we fail to see evidence in the skiagram. Metastatic arthritis comparable to similar diseases elsewhere is by no means uncommon and is a source of severe backache; but perhaps the commonest trouble in these joints is relaxation of the ligaments. Jones and Lovett say that such relaxation is uncommon and they have rarely seen it. With all possible deference to such high authority, I think it is quite common. The outstanding cause for this is childbirth. In ruminants these joints are normally relaxed to such an extent before labour that the cowherd can foretell the approximate date of calving from palpation of them. After birth involution takes place in them pari passu with that of the uterus. Precisely the same phenomenon occurs in women-with this difference, relaxation is less marked; but woman is a biped and her sacro-iliac joints must stand a relatively greater strain than do those of a quadruped. In such cases there is a tendency for the sacrum to fall forwards and downwards, producing a painful tension on the ligaments. Here prevention by adequate rest after labour is better than cure, but in old-standing cases rest will do much and should be insisted upon, treatment being continued by means of a pelvic brace, or better, one of those close-fitting elastic corsets reaching well down on the hips that for some reason or other the Parisians are more expert at making than others.

Tuberculosis is fortunately rare, but is of grave prognosis. Definite tubercle will need years of recumbency and fixation, although operation by bone graft has in recent years much improved the prognosis and shortened the period of treatment. Grafting of this joint, as usually practised, is a difficult operation, consisting in the placing of a graft across the affected joint. Frequently in doing this an abscess is opened, and although this is not a contraindication to the operation it is certainly a complication. Acting on the suggestion of my assistant, M.r. Fleming, recently, in cases both of tubercle and of simple metastatic arthritis, I have adopted the plan of placing a long bone graft from the tibia through both posterior superior spines of the ilium, the graft resting on a prepared bed in the sacrum under the erector spinae muscles. Such a graft is easily inserted, requires no additional fixation, and to my mind represents a sound mechanical principle-that of the tiebeam. Weight is transmitted downwards along the vertebral column and is resolved to a certain extent laterally through the sacro-iliacs, the graft having therefore the same effect as do those iron bars with $\mathbf{X}$-shaped pieces at the end that are customarily seen on houses which have become insecure. This procedure will shorten the period of treatment in tuberculous cases, and in others severe enough to need operation causes relief as soon as the graft has united, and (so patients tell me) prevents that feeling of insecurity which is so terrible and which is the cause of the neurasthenia so commonly associated with sacro-iliac weakness.

\section{Sacralization of the Fifth Lumbar Vertebra.}

We are again indebted to America for observation of this defect. High authorities ${ }^{1}$ tell us that, although it occurs, it is rarely responsible for symptoms. With this I agree, but when it does cause symptoms they are very scvere. In a certain number of individuals the fifth lumbar vertebra is fused to the sacrum, giving four lumbar and six sacral rertebrae. This in itself does no harm, and is found cut by accident in a skiagram. In some cases, however (sometimes combined with fusion of the bodies and sometimes without), the transverse processes of the fifth lumbar vertebra take on sacral characters and impinge on the ilium, forming a joint. The condition may be vcry painful; it can be diagnosed only by $x$ rays, and a good stereoscopic skiagram is necessary before we can pronounce it certainly present. It may cause no symptoms at all, but if symptoms occur they consist in intractable pain, both local and referred to the area supplied by the fourth lumbar nerve, as the junction between the fourth and fifth lumbar nerves lies immediately anterior to the process. Treatment consists in removal of the process, and the proximity of the nerve junction and the depth of wound necessary if the process is approached directly from the back render the operation difficult. It is far better to approach the process by cutting a window from the iliac crest cpposite it, as this serves the double purpose of removing the impinging process and the structure on which it impinges, and is incidentally a relatively easy operation. We will not delay longer with this moderately rare condition; it will suffice if we realize the possibility of its occurrence.

\section{Referred Pain.}

I have already referred to the sympathetic system, and we have seen that it is probably the channel of control of the postural activity of the skeletal muscles; Sherrington maintains that the tone of hollow viscera, controlled by the sympathetic, is in reality postural activity. In addition, nutrition is controlled through the vasomotor fibres of the sympathetic. But the sympathetic contains also the sensory nerves of the viscera. The gangliated cord is connected with the spinal nerves by white rami communicantes, which arise from the anterior primary divisions from the second dorsal to the second lumbar nerves, and from the third (sometimes second or fourth) sacral. These rami obtain from the ventral root motor and secretory fibres and from the dorsal root the sensory nerves for the viscera. These fibres are said to occur not only in the white rami, but to be capable of reaching the sympathetic chain by way of the grey rami. By these grey rami the axons of sympathetic cells reach each of the spinal nerves, and it is $k y^{\circ}$ them that postural and vasomotor control of the body is maintained. For the purpose of this lecture the upper part of the sympathetic does not concern us.

It is clear that, as the senso:y nerves to the viscera are the axons of spinal ganglion cells, pain derived from the viscera niay be referred to the somatic area served by these same ganglia, and it is often so. Study of cases combined with a consideration of anatomy will often enable us to diagnose the source of trouble. A fow examples will suffice to illustrate this.

'The lesser splancinnic nerve from the ninth and tenth dorsal ganglia reaches the solar plexus and thence through the superior mesenteric plexus reaches the intestine as far as the caecum. Pain from this origin may be felt in the lower dorsal and upper lumbar regions, down over the crest of the ilium and in front. The least splanchnic nerve from the last dorsal ganglion reaches the renal plexus, and pain from the kidney may follow the distribution of the twelfth dorsal nerve. Fibres from the first two lumbar nerves pass by the aortic and inferior mesenteric plexuses to the colon, ctc., and an overloaded colon, in addition to the backache it may cause by its mechanical weight and by the toxins it may cause to enter the circulation, may also cause backache (both lumbar and in the buttocks) by pain referred along the distribution of these two nerves.

In the sacral region refered pain is common. The upper sacral nerves communicate directly with the pelvic plexuses, and pain is referred from the utcrus, hladder, prosta', etc. 
This pain is commonly felt over the sacro-iliac joints, but 1 would again emphasize that it is not a deep pain, and a little practice will easily enable us to discriminate between this and the deep tenderness associated with pain on lateral compression of the pelvis which is characteristic of sacroiiiac disalibities. It would seem that by calling attention to such a multiplicity of possible causes of lumbo-sacral backache 1 have but added to the difficulties of the subject, but these cases are very trying and often the trouble is of very long duration. By realizing the possible causes we may often by systematic examination and exclusion reach the correct solution at once, or, even if we do not do so, we may appreciate the fact that the pain is due to several of these causes combined in a vicious circle, and we may attack the different parts of that circle seriatim and achieve a cure.

To summarize briefly : in examining a case of lumbo-sacral backache first exclude gross disease, injury, and arthritis, and have a skiagram taken. Then look for outside mechanical causes and outside causes of overstrain of the nervous system. Finally, remember the paramount importance of the sympathetic system and seeli for referred pain.

Treatment has been briefly indicated in each case, but the ralue of mobilization in suitable cases must be remembered, and this valuable therapeutic measure should not be relegated to outsiders.

REFEREACES.

1 Jones and Lovett: Orthop-xedic Surgery. Deformities, British Medical Jocrnal, April, 1921.

\section{ACUTE DIABETES FOLLOWING MUMPS. BY}

ADAM PATRICK, M.D., M.R.C.P.

PROFESSOR OF MEDICINE, UNIVERSITY OF ST. ANDREWS; VISITING PHYSICIAN, DUNDEE ROYAL INFIR:MARY.

THE occurrence of pancreatitis as a complication in the course of an attack of mumps is well known, and has frequently been described. Farnam ${ }^{1}$ in 1922 collected 119 such cases fromi the literature, and added one of her own, in which laparotomy was performed. She summarizes the case records thus:

“An acute abdominal condition, probably pancreatitis, is sometimes associated with epidemic parotitis. Ii cocurs more often in boys and young men than in other classes of the population; it usually follows, but may precede, the parotitis, or may be the only manifestation of the disease. It is characterized by intense epigastric pain, of ten vomiting, occasionally diarrhoea or constipation, and a slight rise in temperature. A mass may sometimes be felt in the epigastrium. It usually runs a short benign course, and has not been known to affect the internal secretion of the pancreas."

All these 119 patients, with one exception, recovered. It may be stated that the only cases in which the pancreas has actually been seen at this stage are Farnam's (at operation) and Lemoine and Lapasset's ${ }^{2}$ (post mortem). In the former case the pancreas was found to be red and tense, with three probable areas of fat necrosis, and the impression gained was one of acute pancreatitis.

Lemoine and Lapasset's case was that of an Algerian soldier who was admitted to hospital with mumps on October 2nd, 1902 . The attack was at first simple, but on the tenth day there occurred shivering with - high fever, and swelling of the testicle. By the fourteenth day the temperature had fallen to normal, and the patient seemed to be well enough for discharge from hospital. Next day, how ever, he complained of having slept badly, and had vomited. The temperature was normal, but there was epigastric tenderness and the spleen was palpable. Jaundice and more vomiting followed, and he died on October 18th, after an attack of haernatemesis. At the post-mortcm examination the gall bladder was found to be swollen and oedematous, apparently from pressure by an enlarged gland on the common duct. The pancreas had almost tripled its normal weight (190 grams), and was oedematous and congested, with swelling of the lymph glands round about: and congested, with swelling of the ymph glands round about: acini and epithelial cells, with diminution in size of the islets of Langerhans, through pressure of the swollen ducts:

Although symptoms of pancreatitis are thus relatively common in the course of mumps, glycosuria or diabetes has seldom been described, and Garrod ${ }^{3}$ in his Lettsomian lectures (1912) commented on the rarity of glycosuria in this connexion. I have been able to find records of only three cases in the literature, with a doubtful fourth, and $I$ add a brief description of a fifth case, a very acute one, which recently came under my own observation.

1. Harris ${ }^{4}$ (1899) describes the case of a farmer, aged 42 , who three years previously had had an attack of mumps. A month after recovery from this attack polyuria had set in; thirst, hunger, after recovery from this attack polyuria had set in; thirst, hunger,
and loss of weight followed, and these symiptoms had continued and loss of weight followed, and these symiptoms had continued
with gradually increasing severity. When Harris saw him he was with gradually increasing severity. When Harris saw him he was
emaciated, having lost $30 \mathrm{lb}$. in weight, and the knee-jerks were emaciated, having lost $30 \mathrm{lb}$. in weight, and the knee-jerks were
absent; 6,440 c.cm. of urine, containing 4.6 per cent. of glucose, were passed in the day.

2. Barbieri ${ }^{5}$ (1903) mentions the oase of a boy, aged 6, who contracted mumps during an epidemic. Six days after the onset of the symptoms he complained of continuous pain above the umbilicus which radiated round the right side to the right lumbar region. The pain was attributed by the parents to worms, and the child was dosed with calomel and santonin. The abdominal pain continued, and next day there were also vomiting and diarrhoea. After two days of this the boy developed a voracious appetite and great thirst, but cverything he ate was vomited an hour later. He drank 2 litres of water in a day. Barbieri was now called to see the patient, and found that he had slight epigastric tenderness, while the faeces under the microscope showed many droplets of fat. The urine had a specific gravity of 1034 and contained sugar; 2,400 c.cm. were passed in twenty-four hours. The symptoms subsided gradual the arine was sugar-free, the specific gravity being 1015 and the daily amount no more than 600 c.cm.

3. Gilhespy and Holden ${ }^{6}$ (1917) record the case of a lad, aged 16, who in May, 1916, had an attack of mumps, without testicular or abdominal symptoms. On the third or fourth day of this illness the patient became very thirsty, and began to pass a large quantity of urine. When scen in hospital he showed pronounced signs of diabetes mellitus, with sugar and acetone bodies in the urine. Tuberculosis of the lungs also was present, and he died in five months. The authors mention that at the post-mortem show hyaline degeneration.

4. The case which I have classed as doubtful was described by Routh $^{7}$ in 1912. Here a man, aged 58, on April 13th developed a right-sided parotid swelling which was attributed to a chill experienced five days before. Two superficial ulcers were present on the right side of the tongue. Some pyrexia followed and $\mathrm{nx}$ -
tension of the swelling to the submaxillary region. By April $28 \mathrm{th}$ the swelling was more severe, and incision under an anaesthetic the swelling was more severe, and incision was found to contain was contemplated. On and aceto-acetic acid (specific gravity 1058). By May 1st sugar and aceto-acetic acid (specific gravity the aceto-acetic acid had disappeared, and on to have been one was free from sugar. This case would seem not to have been one
of mumps, but rather one in which glycosuria was associated with septic parotitis.

I shall now describe a case in which diabetes followed mumps and ran a rapid course to death, the patient coming under my care in the last stage.

5. E. A., a girl aged 8, was admitted to Dundee Royal Infirmary on December 2nd, 1923, in coma. The history was that five weeks previously she had developed mumps, which ran an ordinary course, previously she there had been no complaint of abdominal pain. Two weeks after the attack of mumps came on she began to pass a large quantiiy of urine, and had to get up sometimes eight a large quanticy of urine, and the course of a night to empty the bladder. She comtimes in the course of a nighed of great thirst, and her appetite increased. After. a weel plained of great thirst, and he other symptoms continued unabated. There was also pronounced constipation. A few days before her There was also pronounced constipation. A new mentally, and on admission to hospital she became confused ndentaly, and onto November 30 th she failed to recognize her friends. She went into coma on Decemiver 1st, and when brought to hospital on December 2nd was deeply unconscious. A specimen of urine obtained by catheterization showed 8 per cent. of glucose and much aceto-acetio acid, and the breath had a strong smell of acetone. In spite of treatment by insulin she

It is interesting to note that although abdominal pain is present in the common type of pancreatitis which complicates mumps, it was absent in Gilhespy and Holden's case, in that which I have described, and it is not referred to as having occurred in Harris's, the only three cases of mumps among those recorded in which true diabetes seems to have developed.

\section{REFERFNCES.}

1 Farnam: Amer. Journ. Mell. Sci. 1922, cixi1i, 859. 2 Lemoine and ret. 640.

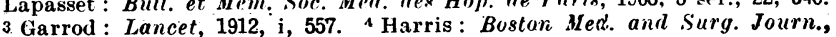
1899, exl, 465, 5 Barbieri: Gazz. degli Osped., 1j09, xxx, 273. 'Gilhespy and Holden: British Medical Jouknal, 1917, ii, 115. ' Routh: BRITIsH MEDICAL JourNal, 1912. ii, 64 . 